\title{
Dieta do tucunaré-amarelo Cichla monoculus (Bloch \& Schneider) (Osteichthyes, Cichlidae), no Reservatório de Lajes, Rio de Janeiro, Brasil
}

\author{
Luciano Neves dos Santos ${ }^{1}$ \\ Alejandra Filippo Gonzalez ${ }^{1}$ \\ Francisco Gerson Araújo ${ }^{1}$
}

\begin{abstract}
Diet of Cichla monoculus (Bloch \& Schneider) (Osteichthyes, Cichlidae) in Lajes' Reservoir, Rio de Janeiro, Brazil. The diet of Cichla monoculus (Bloch \& Schneider, 1801) in Lajes's Reservoir, a major impoundment in Rio de Janeiro State, Brazil, was assessed, from fishes collected in 1994, 1996 and 1999/2000. Gut contents in individuals was analyzed by the index of relative importance (IRI) which deals with numerical, gravimetrical and frequency of occurrence. Cichla monoculus showed a strong piscivorous habits feeding on Cichlidae, Characidae and Pimelodidae, in decreasing order of importance, with a remarkable cannibalism on young-of-the-year. Others minor items in the diet were Macrobrachium sp. and Odonata. Changes in feeding composition varied with reservoir's zones and seasons, with higher diversity in Autumn and peaks of cannibalism in lower zone during Spring/Summer. Overall, only one third of fish species composition in the reservoir are predated by $C$. monoculus. Condition factor $(\mathrm{k})$ and fullness index varied closely with higher values in lower zone, and lower records in Winter.

KEY WORDS. Cichla, trofic ecology, diet, reservoirs, freshwater fish, condition factor, feeding habits
\end{abstract}

As espécies do gênero Cichla (Schneider, 1801), também conhecidas como tucunarés, são naturais da Bacia Amazônica e representam o principal grupo de peixes piscívoros da família Cichlidae na América do Sul (GoLDSTEIN 1973; Lowe-MCCONNEL 1975). O tucunaré-amarelo, Cichla monoculus (Bloch \& Schneider, 1801), apresenta ampla distribuição pelo território nacional, uma vez que foi introduzido em inúmeros açudes e represas do país visando o incremento da pesca esportiva e da piscicultura extensiva e semi-extensiva (FONTENELE \& PEIXOto 1979; Braga 1982; Fontenele 1982; PEIXOTO 1982; Oliveira et al. 1986; SANTOS et al. 1994; AGOSTINHO 1994). Pouco é conhecido sobre a ecologia trófica de peixes em represas com Cichla sp., destacando-se o trabalho de WiLliams et al. (1998) na Venezuela. No Brasil, estudos da dieta de peixes têm se restringido a outras espécies carnívoras (GEALH \& HAHN 1998; POMPEU 1999; SANTOS \& FORMAGIO 2000), embora alguns trabalhos tenham abordado aspectos da ecologia trófica de tucunarés Cichla sp. em reservatórios (SANTOS et al. 1994; ARAÚJO-LIMA et al. 1995; SANTOS 1996).

1) Laboratório de Ecologia de Peixes, Posto de Aqüicultura, Universidade Federal Rural do Rio de Janeiro. Antiga Rodovia Rio-São Paulo, Km 47, 23851-970 Seropédica, Rio de Janeiro, Brasil.

E-mail: lucianosantos@hotmail.com; alejandrafilippo@hotmail.com; gerson@ufri.br 
Embora reconhecido como piscívoro voraz (GOLDSTEIN 1973; LOWEMCConNel 1975; PEIXOTO 1982) e apesar da carência de informações sobre o comportamento alimentar da espécie em ambientes represados e de eventuais impactos causados à ictiofauna nativa, C. monoculus foi introduzido no Reservatório de Lajes entre as décadas de 50 e 60 (OLIVEIRA et al. 1986), visando o controle da superpopulação de tilápias e de peixes forrageiros, assim como para o incremento da pesca esportiva. Em geral, a introdução indiscriminada de espécies alóctones e exóticas, sobretudo piscívoras, têm resultado em severos prejuízos a ictiofauna autóctone, devido, principalmente, a atividade predatória da espécie introduzida e/ou pela transmissão de organismos patogênicos (BRITSKI 1994). A introdução do dourado Salminus maxilosus (Schneider, 1940) no rio Paraíba do Sul, por exemplo, uma espécie ictiófaga de grande voracidade, resultou em uma predação intensa sobre juvenis de piabanha Brycon sp., a ponto de reduzir drasticamente sua população naquele ecossistema (CARAMASCHI 1994).

Neste contexto, estudos sobre a ecologia alimentar de peixes são de fundamental importância para o conhecimento autoecológico das espécies, de eventuais interações intra e inter-específicas, bem como para o delineamento da estrutura trófica de toda a comunidade, fornecendo subsídios à implantação de técnicas de cultivo intensivo ou à elaboração de programas de manejo pesqueiro e de conservação (NIKOLSKY 1963; JUNGER et al. 1988; ZAVALA-CAMIM 1996).

O presente trabalho tem por objetivo analisar aspectos da ecologia trófica de C. monoculus no Reservatório de Lajes, a fim de contribuir para o conhecimento da dieta alimentar da espécie, suas variações espaciais e temporais, bem como identificar eventuais interações da espécie com as demais presentes no ambiente.

\section{MATERIAL E MÉTODOS}

\section{Área de estudo}

O Reservatório de Lajes destaca-se como o maior represamento do Estado do Rio de Janeiro, localizando-se nas vertentes da Serra do Mar $\left(22^{\circ} 42^{\prime}-22^{\circ} 50^{\prime}\right.$ 'S; $43^{\circ} 53^{\prime}-44^{\circ} 05^{\prime} \mathrm{W}$ ) entre os municípios de Piraí e Rio Claro. O lago artificial foi formado entre os anos de 1905 e 1908, através das águas desviadas do rio Piraí pelo túnel de Tocos e pelo represamento do Ribeirão das Lajes e de diversos outros tributários de pequeno porte como o rio da Prata e o rio do Pires. A represa ocupa cerca de $30 \mathrm{~km}^{2}$ de superfície ao nível de $415 \mathrm{~m}$ acima do mar, tendo como principal finalidade a geração de energia elétrica pela Light Serviços de Eletricidade S.A., atendendo parte da demanda do Estado. Além disso, o elevado nível de conservação ambiental do Reservatório de Lajes e sua área de entorno, permitiu a manutenção de um excelente padrão de qualidade de águas (oligotróficas) que abastecem parte da exigência do Estado e permite o desenvolvimento de diversas de espécies de peixes e organismos (OliveIRA et al. 1986).

Para comparações espaciais, a represa foi dividida em três zonas: alta compreendendo as cabeceiras da represa, próxima a embocadura dos principais rios e ribeirões que abastecem o reservatório, e que apresenta menores profundidades, margens menos íngremes e freqüentemente colonizada por macrófitas aquáticas; 
intermediária - localizada entre as zonas alta e baixa, é a região mais extensa do corpo da represa, compreendendo as baías mais amplas e de maiores dimensões; baixa - situada nas proximidades da barragem, possuindo margens com relevos abruptos, maiores profundidades, e menores disponibilidades de estruturas submersas e abrigos (Fig. 1).

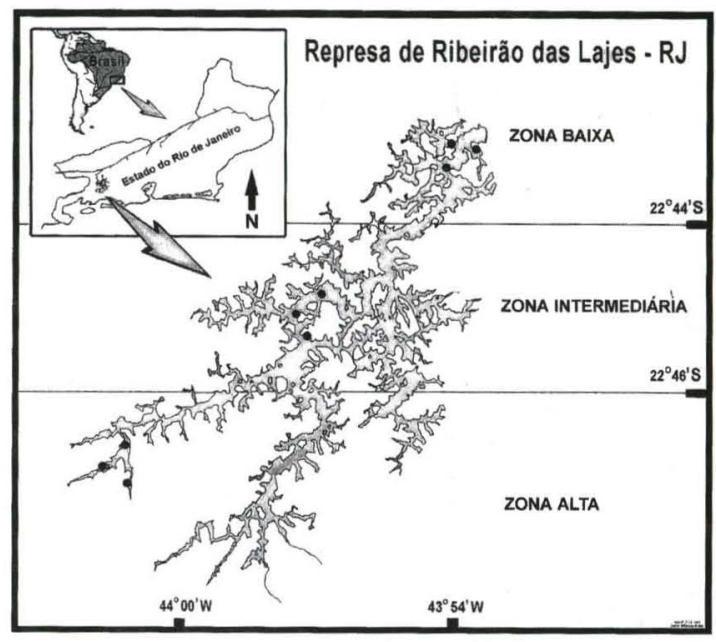

Fig. 1. Mapa do Reservatório de Lajes enfatizando sua localização geográfica e as respectivas divisões espaciais (zonas alta, intermediária e baixa). $(\cdot)$ Locais de coleta nas zonas do Reservatório.

\section{Coleta e análise de dados}

Foram realizadas coletas mensais de peixes no período de janeiro à dezembro de 1994 e 1996, e abril de 1999 à março de 2000, utilizando-se redes de espera com malhas variando entre 2,5 e $5,5 \mathrm{~mm}$ entre nós adjacentes, e tempo médio de permanência de 12 horas. A captura por unidade de esforço (CPUE) foi estabelecida através do número de peixes capturados por $100 \mathrm{~m}^{2}$ de rede por hora. Os peixes capturados foram acondicionados em recipientes isotérmicos com gelo, sendo posteriormente transferidos para o laboratório.

As espécies foram identificadas (ORTEGA \& VARI 1986; FERREIRA et al. 1998), pesadas e medidas em seu comprimento total. O conteúdo estomacal de 87 espécimes de Cichla monoculus foram analisados. Os itens alimentares foram identificados ao nível taxonômico mais baixo possível, sendo posteriormente quantificados em número e peso. Para a análise da dieta alimentar, foi utilizado a freqüência de ocorrência $(\% \mathrm{FO})$, freqüência numérica $(\% \mathrm{FN})$ e freqüência de peso (\%FP), para o cálculo do Índice de Importância Relativa - IIR (PINKAS 1971), onde $\mathrm{IIR}=(\% \mathrm{FN}+\% \mathrm{FP}) \times \mathrm{FO} \%$. A percentagem do IIR foi calculada considerando o valor do IIR para cada item alimentar dividido pelo somatório dos valores de IIR. Foram excluídos os peixes cujos estômagos apresentaram-se vazios. Nas análises 
da participação das espécies de presas consumidas e não consumidas por $C$. monoculus, utilizou-se o valor percentual da CPUE para cada espécie em particular. O índice de repleção (IR) foi estimado visualmente (HYSLOP 1980), através do volume em percentual ocupado pelos itens alimentares nos estômagos.

O fator de condição $(K)$ foi obtido através da seguinte equação (LE CREN 1958): $\mathrm{K}=\mathrm{Pt} / \mathrm{Ct}^{\mathrm{b}}$, onde $\mathrm{Pt}=$ peso total do peixe $(\mathrm{g}) ; \mathrm{Ct}=$ comprimento total do peixe $(\mathrm{mm}) ; \mathrm{e} b=$ coeficiente angular da regressão linear entre $\log _{10} \mathrm{Pt} / \log _{10} \mathrm{Ct}$.

Para a análise sazonal da dieta, os dados foram agrupados em três estações: primavera-verão: setembro à fevereiro; outono: março à maio; e inverno: junho à agosto. A análise espacial, foi feita através da comparação entre as três zonas do reservatório (alta, intermediária e baixa). Análise de variância (ANOVA - Oneway) foi empregada a fim de identificar eventuais diferenças espaciais e temporais para o fator de condição (K) e o índice de repleção (IR).

\section{RESULTADOS}

\section{Dieta alimentar}

Os espécimes de C. monoculus analisados apresentaram comprimento total entre 160 e $530 \mathrm{~mm}$ (média de $323 \mathrm{~mm}$ ) e peso total entre 62 e $1860 \mathrm{~g}$ (média de $513 \mathrm{~g}$ ). Trinta e nove dos 87 (44,8\%) estômagos examinados continham alimento. A dieta de $C$. monoculus consistiu basicamente de quatro categorias principais: peixes, crustáceos, insetos e resíduos orgânicos. Em geral, o item peixes (Tab. I, espécies consumidas) foi a categoria alimentar dominante, participando com $86,6 \%$ no IIR, ocorrendo em 21 (47,0\%) dos estômagos com alimento e representando $69,6 \%$ do número (FN) e $91,8 \%$ peso (FP) totais. Crustáceos e insetos apresentaram pequenas participações na dieta, contribuindo respectivamente com 0,4 e $0,3 \%$ IIR; 7,0 e 4,0\% FO; 3,3 e 6,5\% FN; e 2,6 e 0,2\% FP. Crustáceos foram representados por Macrobrachium sp. enquanto insetos foram constituídos por ninfas de Odonata. O item resíduos orgânicos, que corresponde ao material orgânico em avançado nível de decomposição pelas enzimas gástricas, ocorreu em 42,0\% dos estômagos com alimento, ocupando $12,7 \%$ do IIR e $20,7 \%$ do número total (Fig. 2a).

Dentro da categoria peixes, restos de peixes, constituído por escamas, espinhas e ossos de peixes, destacou-se como item mais representativo, exibindo valores máximos para \% IIR $(42,0), \% \mathrm{FO}(28,0)$ e $\% \mathrm{FN}(46,9)$. Os peixes do grupo Cichlidae representaram $33,5 \%$ do IIR, sendo que juvenis de C. monoculus destacaram-se como principal presa entre os peixes identificados e segundo item em importância na categoria peixes, contribuindo com $26,5 \%$ do IIR, e apresentando valores de $16,0 \%, 26,6 \%$ e $31,4 \%$ em ocorrência, número e peso respectivamente. Tilapia rendalli (Boulenger, 1896) representou 7,1\% de IIR, em função da participação em ocorrência $(12,0 \%)$ e peso $(16,0 \%)$. Os Characidae e Pimelodidae representaram cada qual com 3,3\% do IIR de peixes, sendo que Oligosarcus hepsetus (Cuvier, 1829) obteve maior representatividade para o primeiro (2,7\%IIR) e Pimelodella eigenmanni (Boulenger, 1891) para o segundo (1,9\%IIR). Peixes não identificados consistiram de presas em elevado grau de degradação, e representaram $17,9 \%$ do IIR; $20,0 \%$ FO e 20,3\% FP da categoria Peixes (Fig. 2b). 


$$
\mathrm{n}=39
$$

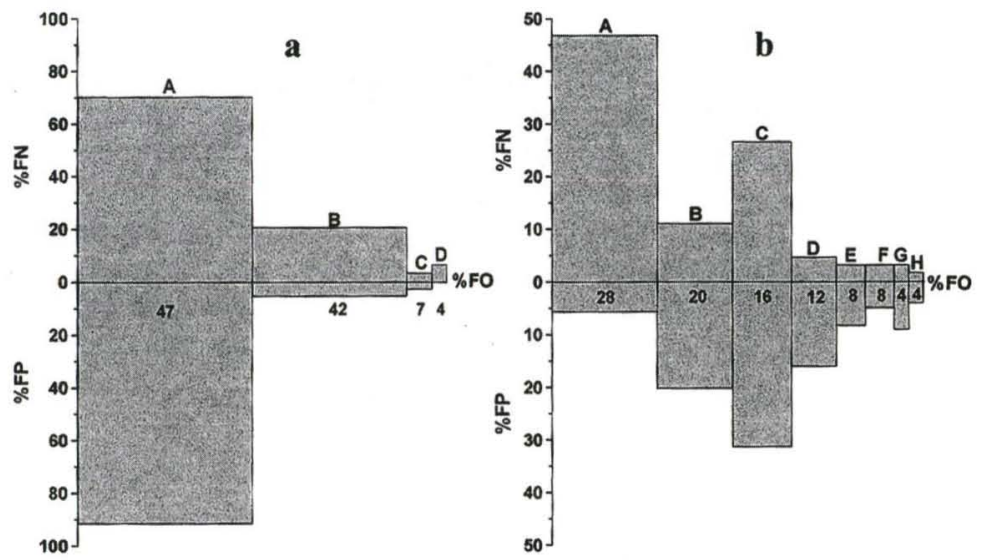

Fig. 2. Indice de Importância Relativa (\% IIR) de C. monoculus por categoria alimentar (a) e itens alimentares para a categoria Peixes (b). Categorias: (A) peixes, (B) resíduos orgânicos, $(C)$ crustáceos, (D) insetos. Itens alimentares: $(A)$ restos de peixes, $(B)$ peixes não identificados, (C) C. monoculus, (D) T. rendalli, (E) O. hepsetus, (F) P. eigenmanni, (G) R. parahybae, (H) Astyanax sp.

Tabela I. Percentual das capturas por unidade de esforço (CPUEn) em número de espécies consumidas e não consumidas por C. monoculus no Reservatório de Lajes.

\begin{tabular}{|c|c|c|c|}
\hline Espécies não consumidas & $\%$ CPUE & Espécies consumidas & $\%$ CPUE \\
\hline Characiformes & 10,4 & Characiformes & 23,1 \\
\hline Erythrinidae & 2,6 & Characidae & 23,1 \\
\hline Hoplias malabaricus (Bloch, 1794) & 2,6 & Astyanax cf. bimaculatus (Linnaeus, 1758) & 14,6 \\
\hline Hoplerythrinus unitaeniatus (Spix, 1829) & $<0,1$ & Astyanax fasciatus parahybae (Eigenmann, 1908) & 4,7 \\
\hline Curimatidae & 2,1 & Oligosarcus hepsetus (Cuvier, 1829) & 3,8 \\
\hline Cyphocharax gilberti (Quoy \& Gaimard, 1824) & 2,1 & Siluriformes & 2,6 \\
\hline Prochilodus scrofa (Steindachner, 1882) & $<0,1$ & Pimelodidae & 2,6 \\
\hline Anostomidae & 0.1 & Pimelodella eigenmanni (Boulenger, 1891) & $<0,1$ \\
\hline Leporinus copelandii (Steindachner, 1875) & 0,1 & Rhamdia parahybae (Steindachner, 1876) & 2,6 \\
\hline Serrasalmidae & 5,6 & Perciformes & 1.9 \\
\hline Metynnis maculatus (Cope, 1878) & 5,3 & Cichlidae & 1,9 \\
\hline Colossoma macropomum (Cuvier, 1818) & 0,3 & Cichla monoculus (Bloch \& Schneider, 1801) & 1,1 \\
\hline Siluriformes & 59,8 & Tilapia rendalli (Boulenger, 1896) & 0,8 \\
\hline Auchenipteridae & 8,8 & & \\
\hline Paracheunipterus striatulus (Steindachner, 1876) & 8,8 & & \\
\hline Loricaridae & 51,0 & & \\
\hline Loricariichthys spixii (Steindachner, 1877) & 49,5 & & \\
\hline Hypostomus affinis (Steindachner, 1876) & 1,4 & & \\
\hline Hartia loricariformes (Steindachner, 1876) & $<0,1$ & & \\
\hline Gymnotiformes & $<0,1$ & & \\
\hline Gymnotidae & $<0,1$ & & \\
\hline Gymnotus carapo (Linnaeus, 1758) & $<0,1$ & & \\
\hline Symbranchiformes & $<0,1$ & & \\
\hline Symbranchidae & $<0,1$ & & \\
\hline Symbranchus marmoratus (Bloch, 1795) & $<0,1$ & & \\
\hline Perciformes & 2,1 & & \\
\hline Cichlidae & 2,1 & & \\
\hline Geophagus brasiliensis (Quoy \& Gaimard, 1824) & 2,1 & & \\
\hline TotalTotal & 72,3 & & 27,6 \\
\hline
\end{tabular}




\section{Variações espaciais na dieta}

Diferenças na dieta alimentar de C. monoculus foram identificadas entre as zonas da represa. $\mathrm{O}$ item peixes destacou-se como categoria principal da dieta em todas as zonas do reservatório, contribuindo com $74,1 \%$ e $60,5 \%$ do IIR nas zonas intermediária e alta respectivamente, e atingindo o máximo de $98,1 \%$ do IIR na zona baixa. Crustáceos ocorreram nas zonas alta e baixa, contribuindo com 7,4\% do IIR na primeira, e exibindo contribuição praticamente nula na segunda $(0,2 \%$ IIR). Insetos ocorreram exclusivamente na zona intermediária, alcançando 1,8\% IIR de participação. $\mathrm{O}$ item resíduos orgânicos contribuiu expressivamente nas zonas alta e intermediária, com 32,1 e 24,1\%IIR respectivamente, apresentando participação pouco significante (1,8\%IIR) na zona baixa (Fig. 3a).

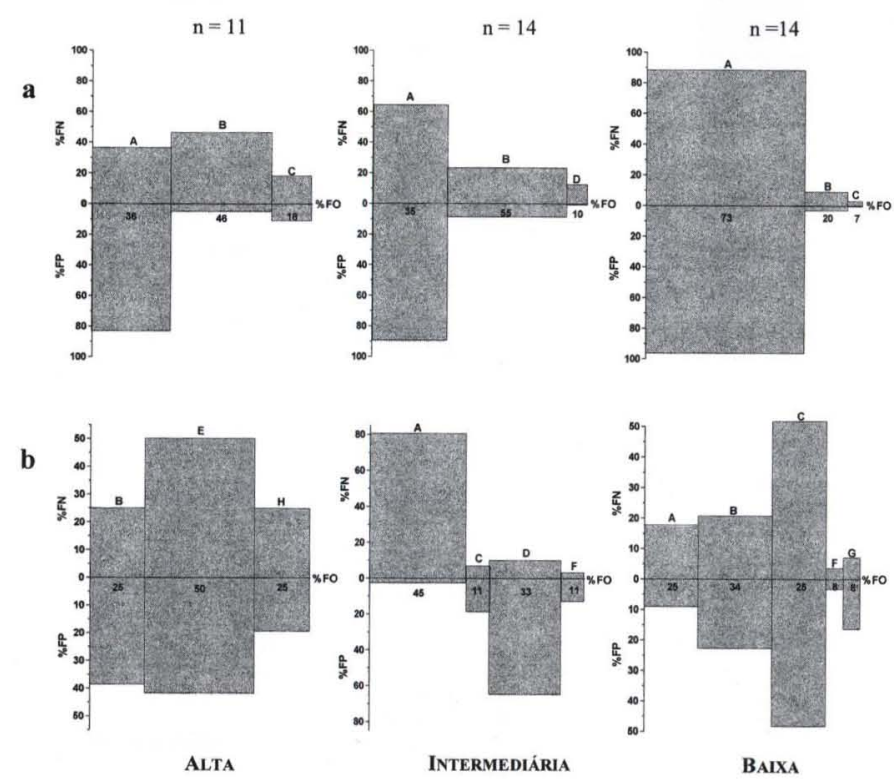

Fig. 3. Indice de Importância Relativa (\% IIR) de C. monoculus por categoria alimentar (a) e itens alimentares para a categoria Peixes $(b)$. Categorias: $(A)$ peixes, $(B)$ resíduos orgânicos, $(C)$ crustáceos, $(D)$ insetos. Itens alimentares: $(A)$ restos de peixes, $(B)$ peixes não identificados, (C) C. monoculus, (D) T. rendalli, (E) O. hepsetus, (F) P. eigenmanni, (G) R. parahybae, (H) Astyanax sp.

Em relação à categoria peixes, os Characidae foram presas dominantes na zona alta, contribuindo com $78,2 \%$ do IIR, seguido por peixes não identificados com $21,8 \%$ de IIR e $38,6 \%$ do peso. Entre os Characidae, $O$. hepsetus representou $50,0 \%$ da ocorrência e número totais, e $42,0 \%$ da FP, contribuindo com $63,0 \%$ do IIR na zona alta. Astyanax sp. participou com $25,0 \%$ em ocorrência e número, representando $15,2 \%$ do IIR na zona alta. Na zona intermediária, restos de peixes constituiu o item mais importante da dieta, contribuindo com 55,6\% do IIR total, 
em função das elevadas participações em ocorrência $(45,0 \%$ FO) e em número ( $80,6 \%$ FN). T. rendalli representou o segundo item em importância, contribuindo com $37,5 \%$ do IIR, $65,4 \%$ de FP e $33,0 \%$ de FO. Canibalismo foi verificado na zona intermediária, sendo que juvenis de tucunaré representaram $4,2 \%$ do IIR e $18,7 \%$ do peso. Os Pimelodidae foram representados exclusivamente por P. eigenmanni, exibindo $2,7 \%$ de IIR, com pequenas participações em ocorrência e número. $\mathrm{Na}$ zona baixa, o canibalismo foi mais significativo (51,6\% IIR), onde juvenis de $C$. monoculus ocorreram em $25,0 \%$ dos estômagos com alimento e representaram $51,7 \%$ e $48,4 \%$ do número e peso respectivamente. Peixes não identificados foram o segundo item em importância nesta zona, ocorrendo com maior freqüência (34,0\%FO) e ocupando $29,7 \%$ do IIR. Restos de peixes ocorreram em $25,0 \%$ dos estômagos com alimento, contribuindo com 13,5\% de IIR. Os Pimelodidae, representados por Rhamdia parahybae (Steindachner, 1876) e P. eigenmani, contribuíram com $5,2 \%$ do IIR na zona baixa, sendo que a primeira espécie participou com $4,0 \%$ do IIR e $16,6 \%$ do peso (Fig. $3 b$ ).

\section{Variações temporais na dieta}

Peixes foi a categoria preferencial na dieta de C. monoculus em todas as estações do ano, contribuindo com cerca de $90,0 \%$ do IIR tanto na primavera-verão como no outono, e decrescendo para 54,6\% do IIR no inverno. Resíduos orgânicos representaram $38,1 \%$ do IIR no inverno, com valores próximos a $12,0 \%$ na primavera-verão e $8,0 \%$ no outono respectivamente. Crustáceos ocorreram no inverno e na primavera-verão, participando com $7,3 \%$ de IIR no primeiro e contribuindo pouco significativamente no segundo $(0,3 \%$ IIR). Insetos ocorreram apenas no outono, representando 2,4\% do IIR total do período (Fig. 4a).

Em relação à categoria peixes, verificou-se canibalismo significativo na primavera-verão, onde juvenis de C. monoculus constituíram item predominante, representando $73,7 \%$ e $55,2 \%$ do número e peso, ocorrendo em $29,0 \%$ dos estômagos com alimento, e exibindo $59,2 \%$ de participação no IIR do período. Peixes não identificados ocuparam a segunda posição em importância na dieta de $C$. monoculus na primavera-verão, ocorrendo em $43,0 \%$ dos estômagos e representando $37,4 \%$ de FP e $36,6 \%$ de IIR respectivamente. $P$. eigenmanni e restos de peixes obtiveram participações reduzidas, representando respectivamente apenas 2,2\% e 1,9\% do IIR no período. O outono foi a estação de maior diversidade de presas, ocorrendo todas as espécies de peixes identificadas. Cichlidae constituíram o principal grupo de peixes consumidos (56,7\% IIR), sendo que $T$. rendalli foi o principal item em peso e ocorrência (30,6\% e $23,0 \%$ respectivamente), contribuindo com $39,2 \%$ do IIR total no período. Canibalismo também ocorreu no outono, quando juvenis de $C$. monoculus ocorreram em 9,5\% dos estômagos e representaram 17,6\% do número e do IIR totais na estação. Os Characidae constituíram o segundo grupo em importância $(18,8 \%$ IIR), sendo que $O$. hepsetus foi a principal espécie, representando $15,2 \%$ do IIR e $16,3 \%$ do peso. Os Pimelodidae representaram apenas $11,2 \%$ do IIR, sendo que $R$. parahybae foi a principal espécie do grupo ( $8,0 \%$ IIR), ocupando a segunda posição em peso $(17,6 \% \mathrm{FP})$ em relação às demais espécies. Peixes não identificados e restos de peixes apresentaram baixas participações no período, representando 6,5 e $6,8 \%$ do IIR respectivamente. O inverno foi o período 
de menor variabilidade de itens na dieta de C. monoculus, sendo representado apenas por restos de peixes e peixes não identificados. Restos de peixes constituiu o item de maior importância no período, representando $97,0 \%$ do IIR, em função de contribuições significativas em número $(96,4 \% \mathrm{FN})$, peso $(81,3 \% \mathrm{FP})$ e em ocorrência $(80 \%$ FO). Os peixes não identificados apresentaram uma participação pouco expressiva (3,0\% IIR), com pequenas contribuições individuais de ocorrência ( $20 \%$ $\mathrm{FO})$, peso (18,7\% FN) e número $(3,6 \% \mathrm{FN})$ (Fig. $4 \mathrm{~b})$.

$$
\mathrm{n}=12 \quad \mathrm{n}=15 \quad \mathrm{n}=12
$$
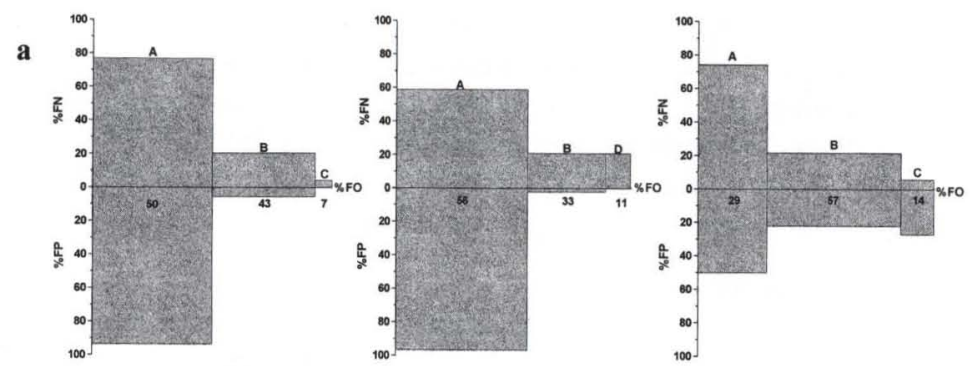

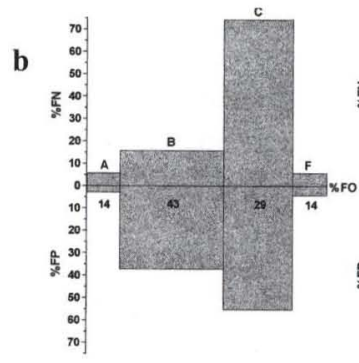

PrimaVera - VerÃo

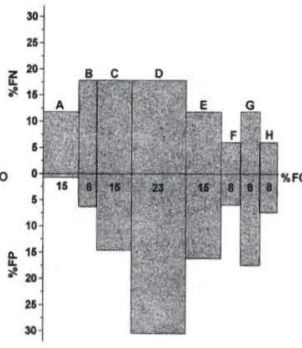

OUTONo

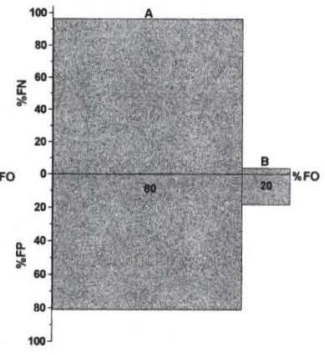

INVERNO

Fig. 4. Indice de Importância Relativa (\% IIR) de C. monoculus por categoria alimentar (a) e itens alimentares para a categoria Peixes (b). Categorias: $(A)$ peixes, $(B)$ resíduos orgânicos, (C) crustáceos, (D) insetos. Itens alimentares: $(A)$ restos de peixes, $(B)$ peixes não identificados, (C) C. monoculus, (D) T. rendalli, (E) O. hepsetus, (F) P. eigenmanni, (G) R. parahybae, (H) Astyanax sp.

\section{Fator de condição e índice de repleção}

O fator de condição (K) variou de 0,04441 a 0,88291 , com média de 0,13897 . O índice de repleção (IR) variou de 5\% a 100\%, com média de $38,1 \%$, nos estômagos que continham algum alimento, sendo que o conteúdo estomacal apresentou um máximo de $4,1 \%$ e um mínimo de $0,1 \%$ do peso total do peixe, com valores médios de $0,6 \%$.

Não foram encontradas diferenças significativas $(P>0,05)$ do fator de condição (K) e do índice de repleção (IR) de C. monoculus entre zonas da represa e estações do ano, no entanto algumas variações foram detectadas (Fig. 5). Em geral, 
as zonas alta e intermediária apresentaram menores valores médios para estas variáveis, quando comparados com a zona baixa. Em relação às estações do ano, os valores médios do índice de repleção foram mais elevados na primavera-verão e no outono. Por outro lado, o fator de condição apresentou valores médios mais elevados no outono, com menores valores na primavera-verão e no inverno.

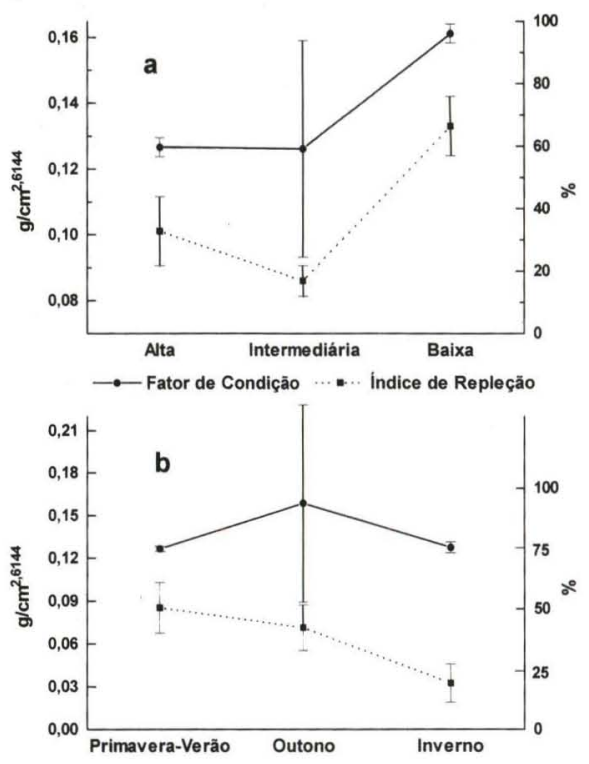

Fig 5. Variações espaciais (a) e temporais (b) do fator de condição e indice de repleção de $C$. monoculus no Reservatório de Lajes.

\section{Relação predador e presas}

O número de presas identificadas nos conteúdos estomacais de C. monoculus representou cerca de $33,3 \%$ do número total de espécies registradas no reservatório, correspondendo a $27,6 \%$ da abundância numérica total de peixes neste ambiente. Astyanax cf. bimaculatus (Linnaeus, 1758), Astyanax fasciatus parahybae (Eigenmann, 1908) e Oligosarcus hepsetus foram as espécies ingeridas que exibiram maiores participações nas capturas, contribuindo com $23,1 \%$ da abundância numérica total.

Entre as espécies não consumidas (Tab. I), os Siluriformes foram representados por quatro espécies: Loricariichthys spixii (Steindachner, 1877), Parauchenipterus striatulus (Steindachner, 1876), Hypostomus affinis (Steindachner, 1876) e Hartia loricariformes (Steindachner, 1876); que contribuíram juntas com $59,7 \%$ da abundância numérica total, sendo que o cascudo-viola L. spixii foi a espécie dominante, contribuindo com $49,5 \%$ do total capturado. Characiformes não consumidos por C. monoculus foram representados por sete espécies: Prochilodus scrofa (Steindachner, 1882), Leporinus copelandii (Steindachner, 1875), Cypho- 
charax gilberti (Quoy \& Gaimard, 1824), Metynnis maculatus (Cope, 1878), Colossoma macropomum (Cuvier, 1818), Hoplerythrinus unitaeniatus (Spix, 1829) e Hoplias malabaricus (Bloch, 1794); que contribuíram juntas com 10,4\% das capturas. Entre os Characiformes não consumidos, M. maculatus, H. malabaricus e $C$. gilberti foram as espécies mais abundantes, contribuindo respectivamente com 5,$3 ; 2,6$ e $2,1 \%$ das capturas totais.

O acará Geophagus brasiliensis (Quoy \& Gaimard, 1824) destacou-se como principal representante da família Cichlidae não consumido, contribuindo com $2,1 \%$ da abundância numérica total. Apesar da ocorrência de canibalismo, C. monoculus apresentou uma pequena participação nas capturas, representando apenas $1,1 \%$ da abundância numérica total.

\section{DISCUSSÃO}

Cichla monoculus apresentou hábito alimentar predominantemente piscívoro no Reservatório de Lajes, com os Cichlidae representando o mais importante grupo consumido, seguidos, em menor proporção, por Characidae e Pimelodidae. Estes resultados são semelhantes aos reportados por WiLliams et al. (1998) no Reservatório de Guri, na Venezuela, para Cichla temensis Humboldt, 1833 e Cichla orinocensis (Humboldt, 1821). No entanto, naquele trabalho, os Cichlidae constituíram o principal grupo de peixes apenas para $C$. orinocensis, enquanto os Characidae representaram o grupo mais importante de peixes na dieta de $C$. temensis. Tais diferenças na dieta foram relacionadas aos locais de alimentação, uma vez que $C$. orinocensis provavelmente alimenta-se em águas mais rasas e com maior disponibilidade de estruturas, onde os Cichlidae de pequeno porte são mais abundantes, enquanto $C$. temensis, especialmente indivíduos maiores, alimentam-se em regiões de águas mais profundas e de habitat menos complexos, com maior abundância de Characidae pelágicos (WILliAMS et al. 1998). Não obstante, SANTOS et al. (1994) observaram que a dieta de adultos de C. monoculus no Reservatório de Marimbondo, bacia do Alto Paraná, foi constituída essencialmente por juvenis de Pimelodidae (mandis) e Cichlidae (acarás), o que coincide com os resultados encontrados no presente trabalho. Por outro lado, РЕIXOTO (1982), registrou uma pequena participação de peixes $(13,0 \%$ FO) nos estômagos de C. ocellaris Bloch \& Schneider, 1801, (= C. monoculus) no açude de Lima Campos, Ceará, em contraste com a elevada abundância de camarões do gênero Macrobrachium (Bate, 1868), que constituíram o principal alimento $(83,9 \%$ FO). A dieta ictiófaga de C. monoculus no Reservatório de Lajes provavelmente está relacionada à abundância de peixes forrageiros ou de pequenas dimensões, do que à uma preferência alimentar específica, o que foi indicado pela variabilidade da composição da dieta entre as zonas da represa e estações do ano.

As diferenças espaciais na dieta de C. monoculus refletem a disponibilidade de presas em função de características intrínsecas de cada zona em particular. A zona alta, região da represa com maior complexidade em habitats, apresentou uma expressiva dominância de Characidae, uma vez que as espécies deste grupo exibem uma natural preferência por pequenos rios e riachos abundantes na referida zona. Nas demais zonas, intermediária e baixa, os Cichlidae constituíram a principal presa, 
seguidos por Pimelodidae, espécies que habitam regiões marginais, no caso das primeiras, e águas mais profundas, no caso das segundas. Em relação aos demais itens consumidos, apesar das pequenas participações, crustáceos (Macrobrachium sp.) podem constituir uma fonte alimentar alternativa na zona baixa, enquanto Insetos, (ninfas de Odonata), da mesma forma na zona intermediária, sobretudo para exemplares de menor tamanho. PiAnKa (1982), observou, no entanto, que o consumo ótimo de alimento está relacionado à economia de energia pelo predador, refletida em uma estratégia alimentar baseada na busca e captura preferenciais por presas, comparativamente mais energéticas e abundantes em suas rotas alimentares naturais. Este autor, ressalta ainda que, o consumo ótimo em um determinado ambiente, raras vezes ocorre em outro, e que a anatomia do animal é limitante à tática de obtenção do recurso alimentar.

Variações temporais na dieta de C. monoculus também ocorreram. Na primavera-verão, a participação de Cichlidae foi dominante, representada essencialmente por juvenis de tucunaré, seguido de uma pequena contribuição de Pimelodidae, indicando o pico reprodutivo de C. monoculus neste período. No outono, verificou-se uma maior diversidade de espécies ingeridas, com participações mais homogêneas de cada espécie na dieta, refletindo um maior compartilhamento e abundância destas espécies no ambiente. No inverno, nenhuma espécie pôde ser identificada, em função do elevado grau de digestibilidade das presas. Neste período, as participações exclusivas de peixes não identificados, e, sobretudo, restos de peixes, estão provavelmente associadas a ofertas reduzidas de alimentos ou à diminuição do metabolismo do predador, refletida na menor taxa de ingestão de presas.

O canibalismo ocorreu durante quase todo o período estudado, sendo mais significativo na primavera-verão, e na zona baixa. na primavera-verão a elevada participação de juvenis de tucunaré na dieta de C. monoculus está provavelmente relacionada ao pico de reprodução da espécie neste período. Na zona baixa o canibalismo pode ser explicado pela menor disponibilidade de abrigos e estruturas no ambiente, resultando em superpredação, e consequentemente favorecendo o aumento da taxa de canibalismo. Não obstante, as taxas de canibalismo podem estar subestimadas, uma vez que os itens resíduos orgânicos, restos de peixes e peixes não identificados podem mascarar a ocorrência real de jovens de $C$. monoculus no estômago de exemplares maiores. Segundo NiKOLSKY (1963), em muitos casos o canibalismo atua para regular a abundância e reduzir o agravamento das relações alimentares que podem ocorrer como resultado das superpopulação, fato que pode estar ocorrendo no Reservatório de Lajes, em função da sua natureza oligotrófica e abundância limitada de espécies forrageiras ou juvenis.

No Reservatório de Lajes, $C$. monoculus apresentou maiores valores para o fator de condição do que C. orinocensis e C. temensis no Lago Guri (WILLIAMS et al. 1998), um reservatório hiperoligotrófico. A melhor condição de C. monoculus no Reservatório de Lajes, poderia estar refletindo uma maior disponibilidade de presas neste ambiente, o que explicaria tais diferenças. Segundo SANTOS \& Formagio (2000), a elevada abundância de recursos alimentares como os camarões do gênero Macrobrachium, em reservatórios nordestinos, e peixes como a sardela Hypophthalmus edentatus (Spix, 1829), no reservatório de Itaipu, provavelmente reduz a intensi- 
dade de predação de piscívoros sobre a ictiofauna de pequeno porte deste ambientes. A elevada abundância de juvenis de C. monoculus no Reservatório de Lajes, indicada pelas altas taxas de canibalismo observadas, poderia estar promovendo um efeito semelhante ao da sardela em Itaipu, ao menos durante determinadas épocas do ano, como foi constatado neste estudo para a primavera-verão.

O número total de presas apresentou uma pequena participação na riqueza e abundância totais do Reservatório de Lajes. Estas participações podem estar relacionadas a composição específica da ictiofauna, uma vez que a maioria das espécies pertencem ao grupo Siluriformes, peixes que apresentam poderosos acúleos nas nadadeiras (e.g. Parauchenipterus striatulus, Pimelodella eigenmanni, e Rhamdia parahybae) e/ou placas ósseas robustas recobrindo o corpo (e.g. Loricarichthyes spixii, Hypostomus affinis e Hartia loricariformes), que inibem a predação sobre estes peixes.

Entretanto, a introdução e o estabelecimento de C. monoculus no Reservatório de Lajes também pode ter resultado em alterações significativas na composição e estrutura da ictiofauna. Embora pouco se conheça sobre os efeitos da introdução de Cichla sp. à comunidade de peixes de ambientes represados, SANTOS (1996), constatou uma redução gradativa do número geral de espécies de peixes do reservatório de Samuel, em Rondônia, relacionado a um aumento na abundância de várias espécies carnívoras, entre elas o tucunaré C. monoculus, indicando uma desestruturação na composição da ictiofauna daquele ambiente. RIVAS \& LUIS (1973) apud FonTENEle \& PEIXoto (1979) e FonTEnEle et al. (1983), reportam, respectivamente, o controle das populações de piranhas Serrasalmus sp. na represa de Las Majaguas (Venezuela) e em açudes e represas da região Nordeste do Brasil através da introdução de tucunarés Cichla sp. PEIXOTO (1982), confirmou a prática de canibalismo por tucunarés $C$. ocellaris e $C$. temensis no açude Lima Campos (Ceará), embora não tenha observado uma predação intensa sobre outras espécies ícticas. De acordo com SANTOS et al. (1994), o estabelecimento do tucunaré C. monoculus e da corvina Plagioscion squamosissimus (Heckel, 1940) no reservatório de Marimbondo, contribuiu para uma simplificação da estrutura ictiofaunística neste ambiente, decorrente da atividade predatória destas espécies. Não obstante, SANTOS \& FoRMAGIO (2000) consideram a introdução da corvina P. squamosissimus com o principal fator responsável pela redução dos estoques ou mesmo extinção das espécies de pequeno e médio porte do reservatório de Volta Grande, na bacia do rio Grande. Desta forma, a pequena participação de forrageiros e de espécies de pequeno porte, as altas taxas de canibalismo e as elevadas abundâncias de Siluriformes constituem evidências de que $C$. monoculus desempenha um papel relevante na estruturação da ictiofauna do Reservatório de Lajes.

AGRADECIMENTOS. Este trabalho recebeu apoio financeiro do Conselho Nacional de Desenvolvimento Científico e Tecnológico (CNPq). A Ligth Serviços de Eletricidade S.A., concessionária do Reservatório, forneceu todo suporte de infra-estrutura, essencial para o desenvolvimento das coletas de campo, no que somos gratos especialmente aos Drs. Sebastiăo Paulino e Ricardo Bichara. Também agradecemos aos estagiários e aos funcionários do Laboratório de Ecologia de Peixes, pela participação nas coletas, triagem das amostras e identificação das espécies.

Revta bras. Zool. 18 (Supl. 1): 191 - 204, 2001 


\section{REFERÊNCIAS BIBLIOGRÁFICAS}

Agostinho, A.A. 1994. Pesquisas, monitoramento e manejo da fauna aquática em empreendimentos hidrelétricos. Semin. Fauna Aquát. e Setor Eletr. Brasil. COMASE, Rio de Janeiro, 1: 38 - 57.

ARAúJo-Lima, C.A.R.M.; A.A. Agostinho; N.N. FABRÉ. 1995. Trophic aspects of fish communities in Brazilian rivers and reservoirs. p. 105-136. In: J.G. Tundisi; C.E.M. BICUDO \& T.M. TuNDISI (Eds). Limnology in Brazil. Rio de Janeiro, ABC/SBL, 376p.

Bragı, R.A. 1982. Crescimento de tucunaré-pinima Cichla temensis Humboldt, em cativeiro (Actinopterygii, Cichlidae). Colet. Trab. Téen. DNOCS, Fortaleza, 2: 101-112.

BRITSKI, H.A. 1994. Sistemática dos peixes de água doce e sua importância nos projetos do setor elétrico. Semin. Fauna Aquát. Setor Eletr. Brasil. COMASE, Rio de Janeiro, 1: 23-30.

Caramaschi, E.P. 1994. Aspectos da distribuição dos Peixes de Água Doce. Semin. Fauna Aquát. Setor Eletr. Brasil. COMASE, Rio de Janeiro, 1: 18-22.

Ferreira, E.J.G.; J.A.S. Zuanon \& G.M. Santos. 1998. Peixes comerciais do médio Amazonas: região de Santarém, Pará. Brasília, Edições Ibama, 214p.

FONTENELE, O. 1982. Contribuição para o estudo da biologia dos tucunarés (Actinopterygii, Cichlidae), em cativeiro - aparelho de reprodução, hábitos de desova e incubação. Colet. de Trab. Téen. DNOCS, Fortaleza, 2: 256-276.

FonTENELE, O. \& J.T. PeiXoto. 1979. Apreciação sobre os resultados da introdução do tucunaré comum, Cichla ocellaris Bloch \& Schneider, 1801, nos açudes do nordeste brasileiro, através da pesca comercial. Bol. Téc. DNOCS, Fortaleza, 37 (2): 109-134.

Fontenele, O.; E.A. VAsconcelos \& E.A. Duarte. 1983. Análise de depleção dos estoques de piranha (Serrasalmus lacépede, 1803) em açudes do Nordeste. Bol. Téc. DNOCS, Fortaleza, 41 (1): 5-26.

Gealh, A.M. \& N.S. Hahn. 1998. Alimentação de Oligosarcus longirostris Menezes \& Gèry (Ostheichthyes, Acestrorhynchinae) do reservatório de Salto Segredo, Paraná, Brasil. Revta bras. Zool. 15 (4): 985-993.

Goldstein, R.J. 1973. Cichlids of the world. New Jersey, T.F.H. Publ. Inc., 382p.

HYSLOP, E.J. 1980. Stomach contents analysis - a review of methods and their application. Jour. Fish. Biol. 17: 411-419.

JUNGER, H.; K. KotRSCHOR \& A. GODSCHIMID. 1988. Situs, relative length and mucosal surface structure of the gut in European cyprinids. Oesterr. Fisch. 41 (5-6): 1-102.

LE CREN, E.D. 1958. Observations on the growth of perch (Perca fluviatilis L.) over twenty-two years with special reference to the effects of temperature and changes in population density. Jour. Anim. Ecol. 27 (2): 287-334.

LOWE-MCCONNELL, R.L. 1975. Fish communities in tropical freshwaters. London, Longman, 337p. NiKolsky, G.V. 1963. The ecology of fishes. London, Academic Press, 352p.

Ol.iveira, S.L. DE; Z.C. Mendes; L.C. Crisóstomo \& F.G. AraúJo. 1986. Resultados preliminares do levantamento ictiológico na represa de Ribeirão das Lajes, estado do Rio de Janeiro. Publ. Avuls Mus. Nac. Rio de Janeiro 65: 87-90.

Ortega, H. \& R.P. VAri. 1986. Annotated checklist of the freshwater fishes of Peru. Smith. Contrib. Zool. 437: 1-25.

Peixoto, J.T. 1982. Alimento de tucunaré, Cichla ocellaris Bloch \& Scheneider, 1801 no açude Lima Campos, Icó, Ceará, (Actinopterygii, Cichlidae). Colet. de Trab. Técn. DNOCS, Fortaleza, 2: $159-172$.

PIANKA, E.R. 1982. Ecologia evolutiva. Barcelona, Omega, 312p.

PINKAS, L. 1971. Food habits study, p. 5-10. In: L. PINKAS; M.S. OLIPHANT; I.L.K. IVERSON (Eds). Food habits of albacore bluefin tuna, and bonito in California waters. Fish. Bull. 152: 1-105.

PomPeU, P.S. 1999. Dieta da pirambeba Serrasalmus brandtii Reinhardt (Teleostei, Characidae) em quatro lagoas marginais do rio São Francisco, Brasil. Revta bras. Zool. 16 (Supl. 2): 19-26.

Santos, G.B. \& P.S. Formagio. 2000. Estrutura da ictiofauna dos reservatórios do rio Grande, com ênfase no estabelecimento de peixes exóticos. Informe Agropec. 203 (21): 98-106. 
Santos, G.B.; P.M. Maia-Barbosa; F. Vieira \& C.M. LóPEZ. 1994. Fish and zooplâncton communities structure in reservoirs of southestern Brazil: effects of the introduction of exotic predatory fish, $p$. 115-132. In: R.M. PINTO-COELHO; A. GIANI \& E. VON SPERLING (Eds). Ecology and human impact on lakes and reservoirs in Minas Gerais with special reference to future development and management strategies. Belo Horizonte, SEGRAC, 435p.

Santos, G.M. 1996. Impactos da Hidrelétrica Samuel sobre as comunidades de peixes do rio Jamari (Rondônia, Brasil). Acta Amazônica 25 (3/4): 247-280.

Williams, J.D. \& K.O. WinEmiLleR; D.C. TAPHORN \& L. BALBAS. 1998. Ecology and status of piscivores in Guri, an oligotrophic tropical reservoir. North Amer. Jour. Fish. Management. 18: 274-285.

ZAVALA-CAMIN, L.A. 1996. Introdução ao estudo sobre alimentação natural em peixes. Maringá, Eduem, 129p.

Recebido em 10.VIII.2000; aceito em 26.VI.2001. 